\title{
REAL MINIMAL HYPERSURFACES IN A COMPLEX PROJECTIVE SPACE
}

\author{
MASAHIRO KON \\ Abstract. A characterization of the geodesic minimal hypersphere in a complex \\ projective space is given.
}

Introduction. Let $C P^{n}$ denote a complex $n$-dimensional projective space equipped with the Fubini-Study metric normalized so that the maximum sectional curvature is 4 . We consider the Hopf fibration $\bar{\pi}$ :

$$
S^{1} \rightarrow S^{2 n+1} \stackrel{\bar{\pi}}{\rightarrow} C P^{n},
$$

where $S^{k}$ denotes the Euclidean sphere of curvature 1. In $S^{2 n+1}$ we have the family of generalized Clifford surfaces whose fibres lie in complex subspaces (see [1]):

$$
M_{2 p+1,2 q+1}=S^{2 p+1}\left(\sqrt{\frac{2 p+1}{2 n}}\right) \times S^{2 q+1}\left(\sqrt{\frac{2 q+1}{2 n}}\right),
$$

where $p+q=n-1$. Then we have a fibration $\pi$ :

$$
S^{1} \rightarrow M_{2 p+1,2 q+1} \stackrel{\pi}{\rightarrow} M_{p, q}^{C}
$$

compatible with $\bar{\pi}$. In the special case $p=0, M_{0, n-1}^{C}$ is called the geodesic minimal hypersphere (see [5]).

In this paper we shall prove the following result.

TheOREM. Let $M$ be a compact orientable real minimal hypersurface of $C^{n}$. If the sectional curvature $K$ of $M$ satisfies $K \geqslant 1 /(2 n-1)$, then $M$ is the geodesic minimal hypersphere $M_{0, n-1}^{C}$.

1. Auxiliary results. Let $M$ be a real hypersurface isometrically immersed in $C P^{n}$. We denote by $J$ the almost complex structure of $C P^{n}$ and by $C$ a unit normal of $M$ in $C P^{n}$. For any vector field $X$ tangent to $M$ we put

$$
J X=P X+f(X) C,
$$

where $P X$ is the tangential part of $J X$ and $f$ is a 1 -form. Then $P$ is an endomorphism on the tangent bundle of $M$. We now put $U=-J C$. Then $U$ is the unit vector field tangent to $M$. From (1.1) we have

$$
P^{2} X=-X+f(X) U, \quad P U=0 .
$$

Received by the editors April 18, 1979 and, in revised form, July 7, 1979.

AMS (MOS) subject classifications (1970). Primary 53B25, 53C40; Secondary 53B35, 53C55.

Key words and phrases. Real hypersurfaces, geodesic minimal hypersphere, sectional curvature. 
The Riemannian metric tensor field of $M$ will be denoted by $g$. Then we obtain

$$
g(P X, Y)+g(X, P Y)=0, \quad f(X)=g(X, U) .
$$

We denote by $\bar{\nabla}$ (resp. $\nabla$ ) the operator of covariant differentiation of $C P^{n}$ (resp. $\nabla)$. Then the Gauss and Weingarten formlas are given respectively by

$$
\bar{\nabla}_{X} Y=\nabla_{X} Y+g(A X, Y) C \text { and } \bar{\nabla}_{X} C=-A X
$$

for any vector fields $X$ and $Y$ tangent to $M$. We call $A$ the second fundamental form of $M$, which can be considered as a symmetric $(2 n-1,2 n-1)$-matrix.

From (1.1) and Gauss and Weingarten formulas we have

$$
\begin{gathered}
\nabla_{X} U=P A X, \\
\left(\nabla_{X} P\right) Y=f(Y) A X-g(A X, Y) U .
\end{gathered}
$$

Let $R$ denote the Riemannian curvature tensor of $M$. Then we have the following Gauss and Codazzi equations:

$$
\begin{gathered}
R(X, Y) Z=g(Y, Z) X-g(X, Z) Y+g(P Y, Z) P X-g(P X, Z) P Y \\
+2 g(X, P Y) P Z+g(A Y, Z) A X-g(A X, Z) A Y \\
\left(\nabla_{X} A\right) Y-\left(\nabla_{Y} A\right) X=f(X) P Y-f(Y) P X+2 g(X, P Y) U .
\end{gathered}
$$

From (1.6) the Ricci tensor $S$ of $M$ is given by

$$
S(X, Y)=2(n-1)+3 g(P X, P Y)+\operatorname{Tr} A g(A X, Y)-g\left(A^{2} X, Y\right),
$$

where $\operatorname{Tr} A$ denotes the trace of $A$. If $\operatorname{Tr} A=0$, then $M$ is said to be minimal.

LEMMA 1. Let $M$ be a real hypersurface of $C P^{n}$. If $M$ is minimal, then

$$
\operatorname{div}\left(\nabla_{U} U\right)=2(n-1)-\operatorname{Tr} A^{2}+\frac{1}{2}|[P, A]|^{2} .
$$

Proof. First of all, we have [6]

$$
\operatorname{div}\left(\nabla_{U} U\right)-\operatorname{div}((\operatorname{div} U) U)=S(U, U)+\frac{1}{2}|L(U) g|^{2}-|\nabla U|^{2}-(\operatorname{div} U)^{2},
$$

where $L(U) g$ denotes the Lie derivative of $g$ with respect to $U$ and || denotes the length with respect to $g$. Since $P$ is skew-symmetric and $A$ is symmetric, (1.4) implies that $\operatorname{div} U=0$ and hence $\operatorname{div}((\operatorname{div} U) U)=0$. Thus

$$
\operatorname{div}\left(\nabla_{U} U\right)=S(U, U)+\frac{1}{2}|L(U) g|^{2}-|\nabla U|^{2} .
$$

On the other hand, from (1.2) and (1.3), we find

$$
|\nabla U|^{2}=\operatorname{Tr} A^{2}-g\left(A^{2} U, U\right) .
$$

From the minimality of $M$ and (1.8) we have

$$
S(U, U)=2(n-1)-g\left(A^{2} U, U\right) .
$$

Substituting these equations into (1.9), we have

$$
\operatorname{div}\left(\nabla_{U} U\right)=2(n-1)-\operatorname{Tr} A^{2}+\frac{1}{2}|L(U) g|^{2} .
$$

From (1.4) we see that

$$
(L(U) g)(X, Y)=g\left(\nabla_{X} U, Y\right)+g\left(\nabla_{Y} U, X\right)=g((P A-A P) X, Y),
$$

from which $|L(U) g|^{2}=|[P, A]|^{2}$, where $[P, A]=P A-A P$. From this and (1.10) we have our equation. 
In the sequel, we compute the Laplacian for the second fundamental form $A$ of $M$ (see [4]).

LeMMA 2. Let $M$ be a real minimal hypersurface of $C P^{n}$. Then

$$
g\left(\nabla^{2} A, A\right)=\sum_{i, j} g\left(\left(R\left(e_{j}, e_{i}\right) A\right) e_{j}, A e_{i}\right)-3 \operatorname{Tr} A^{2}+\frac{3}{2}|[P, A]|^{2},
$$

where $\left\{e_{i}\right\}$ denotes an orthonormal frame for $M$.

Proof. Let $X$ be an arbitrary vector tangent to $M$. Then (1.7) implies

$$
\sum_{j}\left(\nabla_{e_{j}} A\right) e_{j}=0 .
$$

From (1.4), (1.5), (1.7) and (1.11) we obtain

$$
\begin{aligned}
g\left(\nabla^{2} A, A\right) & =\sum_{i, j} g\left(\left(\nabla_{e_{j}} \nabla_{e_{j}} A\right) e_{i}, A e_{i}\right) \\
& =\sum_{i, j} g\left(\left(R\left(e_{j}, e_{i}\right) A\right) e_{j}, A e_{i}\right)-3 g\left(A^{2} U, U\right)+3 \operatorname{Tr}(A P)^{2} .
\end{aligned}
$$

Since $\operatorname{Tr}(A P)^{2}=\operatorname{Tr} A^{2} P^{2}+\frac{1}{2}|[P, A]|^{2}$, we obtain

$$
-3 g\left(A^{2} U, U\right)+3 \operatorname{Tr}(A P)^{2}=-3 \operatorname{Tr} A^{2}+\frac{3}{2}|[P, A]|^{2} .
$$

Substituting (1.13) into (1.12), we have our assertion.

We use the following

LEMMA 3 ([2]). Let $M$ be a real hypersurface of $C P^{n}$. Then we have

$$
|\nabla A|^{2} \geqslant 4(n-1) \text {. }
$$

2. Proof of theorem. Since $M$ is compact orientable, Lemmas 1, 2 and 3 imply

$$
\begin{aligned}
0 & \leqslant \int_{M}\left[|\nabla A|^{2}-4(n-1)+\frac{1}{2}|[P, A]|^{2}\right] * 1 \\
& =\int_{M}\left[\operatorname{Tr} A^{2}-\sum_{i, j} g\left(\left(R\left(e_{j}, e_{i}\right) A\right) e_{j}, A e_{i}\right)\right] * 1,
\end{aligned}
$$

where ${ }^{*} 1$ denotes the volume element of $M$. We choose an orthonormal frame $\left\{e_{i}\right\}$ of $M$ such that $A e_{i}=\lambda_{i} e_{i}(i=1, \ldots, 2 n-1)$. Then

$$
\begin{aligned}
-\sum_{i, j} g\left(\left(R\left(e_{j}, e_{i}\right) A\right) e_{j}, A e_{i}\right) & =\sum_{i, j}\left[g\left(A R\left(e_{j}, e_{i}\right) e_{j}, A e_{i}\right)-g\left(R\left(e_{j}, e_{i}\right) A e_{j}, A e_{i}\right)\right] \\
& =-\frac{1}{2} \sum_{i, j}\left(\lambda_{j}-\lambda_{i}\right)^{2} K_{i j},
\end{aligned}
$$

where $K_{i j}$ denotes the sectional curvature of $M$ spanned by $e_{i}$ and $e_{j}$. In accordance with the assumption, we get $K_{i j} \geqslant 1 /(2 n-1)$ and hence

$$
-\sum_{i, j} g\left(\left(R\left(e_{j}, e_{i}\right) A\right) e_{j}, A e_{i}\right) \leqslant-\frac{1}{2(2 n-1)} \sum_{i, j}\left(\lambda_{j}-\lambda_{i}\right)^{2}=-\operatorname{Tr} A^{2}
$$

Therefore, the right-hand side of (2.1) is nonpositive. Hence we have $|\nabla A|^{2}=$ $4(n-1)$ and $P A=A P$. Applying a theorem of [2] or [3], we see that $M$ is $M_{p, q}^{C}$. On 
the other hand, if $p, q \geqslant 1$, then the sectional curvature $K$ of $M_{p, q}^{C}$ takes value 0 for some plane section. But the sectional curvature $K$ of $M_{0, n-1}^{C}$ satisfies $K>1 /(2 n-$ 1). Consequently, $M$ is the geodesic minimal hypersphere $M_{0, n-1}^{C}$.

\section{REFERENCES}

1. H. B. Lawson, Jr., Local rigidity theorems in rank-1 symmetric spaces, J. Differential Geometry 4 (1970), 349-357.

2. Y. Maeda, On real hypersurfaces of a complex projective space, J. Math. Soc. Japan 28 (1976), 529-540.

3. M. Okumura, On some real hypersurfaces of a complex projective space, Trans. Amer. Math. Soc. 212 (1975), 355-364.

4. J. Simons, Minimal varieties in riemannian manifolds, Ann. of Math. (2) 88 (1968), 62-105.

5. R. Takagi, Real hypersurfaces in a complex projective space with constant principal curvatures, J. Math. Soc. Japan 27 (1975), 43-53.

6. K. Yano, On harmonic and Killing vector fields, Ann. of Math. (2) 55 (1952), 38-45.

Department of Mathematics, Faculty of Education, Hirosaki Universtty, Hirosaku, 036 Japan 